\title{
Cultural Characteristics and Pathogenic Variations among Cochliobolus carbonum Isolates in Yunnan Province of China
}

\author{
Jun-Xiang Zhang1, Yi-Xin Wu², Hon-Hing Ho³, Can-Hua Lu², Yue-Qiu He ${ }^{1,2 *}$ \\ ${ }^{1}$ Faculty of Plant Protection, Yunnan Agricultural University, Kunming, China \\ ${ }^{2}$ Faculty of Agriculture and Biotechnology, Yunnan Agricultural University, Kunming, China \\ ${ }^{3}$ Department of Biology, State University of New York, New Paltz, NY 12561, USA
}

\begin{abstract}
Northern leaf spot, caused by Cochliobolus carbonum, is an important corn disease that is favored by the temperate mountain climate, and is the most important factor limiting the production of corn in Yunnan Province, China. Cultural characteristics and pathogenic diversity of 25 isolates of $C$. carbonum from Yunnan were studied. The isolates showed some variation in colony morphology and sporulation on potato lactose agar (PLA) medium. Pathogenicity test of the isolates on 8 differential corn inbred lines demonstrated that races 2 and 3 of $C$. carbonum caused the greatest losses in maize production in Yunnan. However, lines MU-4 and W-8053 were resistant to all race 3 isolates, whereas line 337 showed resistance response to all race 2 isolates. The pathogencity and virulence of all isolates were studied on 40 cultivars. The virulence varied with the fungal isolates. In general, race 3 isolates were more virulent than race 2 isolates, but 2 isolates of race 2 proved to be highly virulent and could cause great damage to corn. This information will be useful in the selection of cultivars/lines with different resistance genes for use in designing resistant corn breeding programs.
\end{abstract}

Keywords: Bipolaris zeicola; Conidia; Corn; Cultivar; Leaf spot; Virulence

\section{Introduction}

Cochliobolus carbonum Nelson (anamorph, Bipolaris zeicola), an ascomycetous fungus, can cause northern leaf spot (NLS), a ubiquitous foliar disease of corn (maize) and grasses in many regions of the world [1-3]. NLS is one of the important factors limiting the production of corn in Yunnan Province of China due to the lack of resistant cultivars and temperate mountain climate favorable to the disease development, even though it is not considered to be a serious problem in corn production in other countries of the world [4].

C. carbonum can infect the leaf, leaf sheath, husks, and ears of corn, and the differentiation of pathogenic races is based primarily on the lesion characters on host leaves, such as the shape and size of the lesion spot. Race 0 is avirulent on corn and causes only flecks or minute lesions on inoculated leaves [5]. Race 1 produces the HCtoxin and induces oval to circular lesions $(11.4 \times 3.8 \mathrm{~mm})$ with a dark center and dark-watery margins on corn with genotypes homozygous for the $h m 1$ gene [6-8]. Race 2 induces necrotic, dark to brown, oval to irregular lesions $(1.9 \times 0.7 \mathrm{~mm})$ on susceptible maize and chlorotic or necrotic, circular to oval lesions on resistant maize [7], and Race 3 causes linear lesions $(3.5 \times 0.5 \mathrm{~mm})$ on susceptible maize and small oval or short linear chlorotic lesions on resistant cultivars $[7,9,10]$. Race 4 is characterized by circular to oval lesions $(5-10 \mathrm{~mm})$ on maize inbred with a B73 background and low virulence on inbred line W64A [11-13].

Several studies have documented variability in the pathogenicity of the fungus, and different pathotypes have been reported from many countries using a range of differential corn lines [11]. Little is known about the pathogenic complexity of C. carbonum in China, and understanding the pathotypes of the pathogen is essential for the development of appropriate disease management strategies. Determination of both host specificity and genetic diversity in the pathogen population is also prerequisite to breed for durable resistance in corn cultivars.

The objectives of present study were to (1) compare the cultural characteristics of C. carbonum isolates from Yunnan; (2) determine the extent of pathogenic variability among C. carbonum isolates; (3) ascertain races of $C$. carbonum based on pathogenicity tests using eight corn inbred lines from Yunnan.

\section{Materials and Methods}

\section{Pathogen isolation}

Isolates of C. carbonum were obtained from infected plant leaves of different varieties collected from corn fields in 10 states of Yunnan Province, China, in 2010 and 2011. The pathogen was isolated by following standard tissue isolation procedure [14]. Single conidium was located microscopically and picked up by a sterile needle. Each spore was eventually transferred to potato lactose agar (PLA) slants for storage.

\section{Studies of cultural characteristics}

The cultural characteristics of different all isolates were studied on 6-cm diameter PLA plates. The morphology of the colony, mycelia and asexual structures were studied after the plates were incubated for 10 days at $25^{\circ} \mathrm{C}$. Morphological identifications of isolates were based on the description of Nelson [15], and Zhang and Sun [16]. The size and shape of 100 conidia were measured under a light microscope.

${ }^{*}$ Corresponding author: Yue-qiu HE, Faculty of Plant Protection, Yunnan Agricultural University, Kunming 650201, Yunnan, China, Tel: 86-087165228221; Fax: 86-0871-65228532; E-mail: ynfh2007@163.com; heyueqiu1955@hotmail.com

Received: November 07, 2013; Accepted November 28, 2013; Published November 30, 2013

Citation: Zhang JX, Yi-Xin Wu, Hon-Hing Ho, Can-Hua Lu, Yue-Qiu He (2013) Cultural Characteristics and Pathogenic Variations among Cochliobolus carbonum Isolates in Yunnan Province of China. J Plant Pathol Microb 4: 210. doi:10.4172/2157-7471.1000210

Copyright: ( 2013 Zhang JX, et al. This is an open-access article distributed under the terms of the Creative Commons Attribution License, which permits unrestricted use, distribution, and reproduction in any medium, provided the original author and source are credited. 
Citation: Zhang JX, Yi-Xin Wu, Hon-Hing Ho, Can-Hua Lu, Yue-Qiu He (2013) Cultural Characteristics and Pathogenic Variations among Cochliobolus carbonum Isolates in Yunnan Province of China. J Plant Pathol Microb 4: 210. doi:10.4172/2157-7471.1000210

\section{Race identification}

The race identification of isolates was based on the lesion types on 8 inbred corn lines grown and inoculated in the greenhouse. Inoculation method followed that described by Welz and Leonard [8,17] with minor modification. The inoculum was prepared by washing the conidia from a 10-days old 6-cm diameter PLA cultures plate with 10

\begin{tabular}{|c|c|c|c|c|c|}
\hline Isolate & Geographic origin & Host & Hypha color & $\begin{array}{l}\text { Colony } \\
\text { reverve color }\end{array}$ & Conidia production $^{a}$ \\
\hline s3 & Shuangjiang, Lincang city & ND-108 & greyish-green & reddish violet & $8.4 \times 10^{6}$ \\
\hline$s 20$ & Zhenkang, Lincang city & BEJZ & greyish-green & reddish violet & $1.4 \times 10^{6}$ \\
\hline s27 & Yongde, Lincang city & LPZ & greyish-green & greyish-green & $6.4 \times 10^{5}$ \\
\hline s33 & Dali, Dali city & YY-22 & greyish-green & greyish-green & $3.4 \times 10^{5}$ \\
\hline s44 & Jianchuan, Dali city & BY-7 & greyish-green & greyish-green & $1.6 \times 10^{6}$ \\
\hline s46 & Dali, Dali city & $\mathrm{XH}-201$ & greyish-green & greyish-green & $6.3 \times 10^{5}$ \\
\hline s53 & Yangbi, Dali city & ZD-808 & greyish-green & greyish-green & $4.6 \times 10^{6}$ \\
\hline s56 & Yangbi, Dali city & BY-16 & greyish-green & greyish-green & $3.7 \times 10^{6}$ \\
\hline s59 & Lufeng, Chuxiong city & HD-4 & grayish-white & greyish-green & $4.4 \times 10^{5}$ \\
\hline$s 61$ & Longling, Baoshan city & TY-7 & grayish-white & greyish-green & $3.3 \times 10^{6}$ \\
\hline$s 68$ & Yiliang,Chaotong ctiy & BDPZ & grayish-white & greyish-green & $7.8 \times 10^{6}$ \\
\hline s74 & Gucheng, Lijiang city & $X X-201$ & greyish-green & greyish-green & $9.1 \times 10^{5}$ \\
\hline s92 & Hongta, Yuxi City & HD-4 & greyish-green & greyish-green & $1.1 \times 10^{6}$ \\
\hline s111 & Longyang, Baoshan City & BY-2 & greyish-green & greyish-green & $3.3 \times 10^{5}$ \\
\hline s118 & Yuxi, Yuxi City & unknown & grayish-white & reddish violet & $5.7 \times 10^{6}$ \\
\hline s120 & Songming, Kunming city & unknown & grayish-white & reddish violet & $2.3 \times 10^{6}$ \\
\hline s144 & Eryuan, Dali city & DY-8 & grayish-white & reddish violet & $3.0 \times 10^{6}$ \\
\hline s146 & Wenshan, Wenshan ctiy & LD-8 & grayish-white & reddish violet & $1.4 \times 10^{6}$ \\
\hline s149 & Wenshan, Wenshan ctiy & YD-90 & greyish-green & greyish-green & $1.3 \times 10^{5}$ \\
\hline s151 & Zhanyi, Qujing city & unknown & greyish-green & greyish-green & $7.5 \times 10^{6}$ \\
\hline s155 & Zhanyi, Qujing city & unknown & greyish-green & Light red & $1.6 \times 10^{6}$ \\
\hline s156 & Zhanyi, Qujing city & unknown & greyish-green & greyish-green & $3.2 \times 10^{5}$ \\
\hline s157 & Zhanyi, Qujing city & unknown & greyish-green & greyish-green & $3.2 \times 10^{5}$ \\
\hline s159 & Zhanyi, Qujing city & unknown & greyish-green & greyish-green & $5.5 \times 10^{5}$ \\
\hline s167 & Zhanyi, Qujing city & $2-A$ & greyish-green & Light red & $3.3 \times 10^{5}$ \\
\hline
\end{tabular}

$\mathrm{a}=$ number of conidia per 6-mm petridish.

Table 1: Cultural characteristics of isolates of Cochliobolus carbonum on potato lactose agar medium incubated for 10 days at $25^{\circ} \mathrm{C}$.
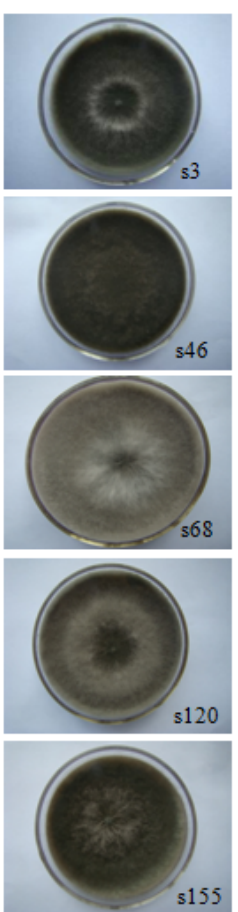
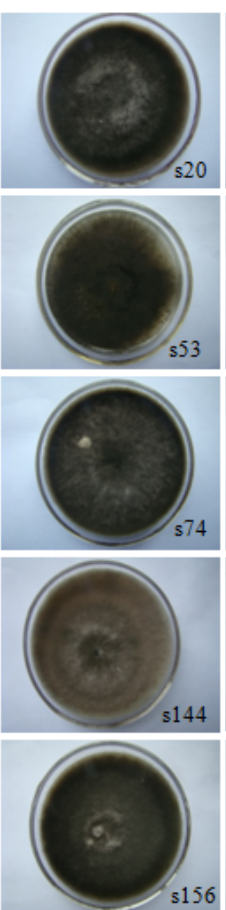
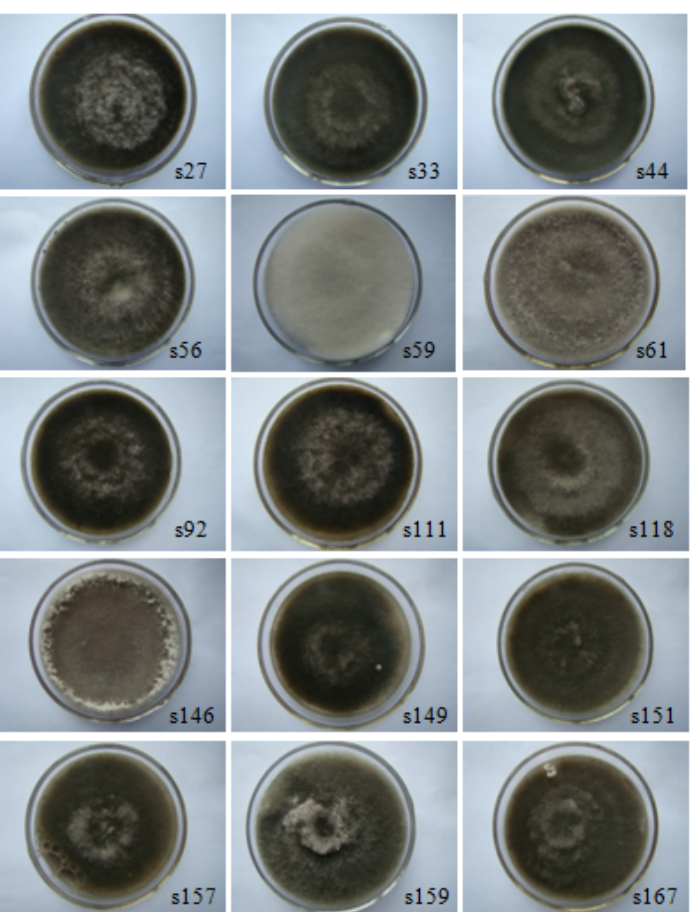

Figure 1: Different cultural characteristics of Cochliobolus carbonum isolates on potato lactose agar (PLA) plates. The isolates number is shown at the bottom right corner. 
Citation: Zhang JX, Yi-Xin Wu, Hon-Hing Ho, Can-Hua Lu, Yue-Qiu He (2013) Cultural Characteristics and Pathogenic Variations among Cochliobolus carbonum Isolates in Yunnan Province of China. J Plant Pathol Microb 4: 210. doi:10.4172/2157-7471.1000210
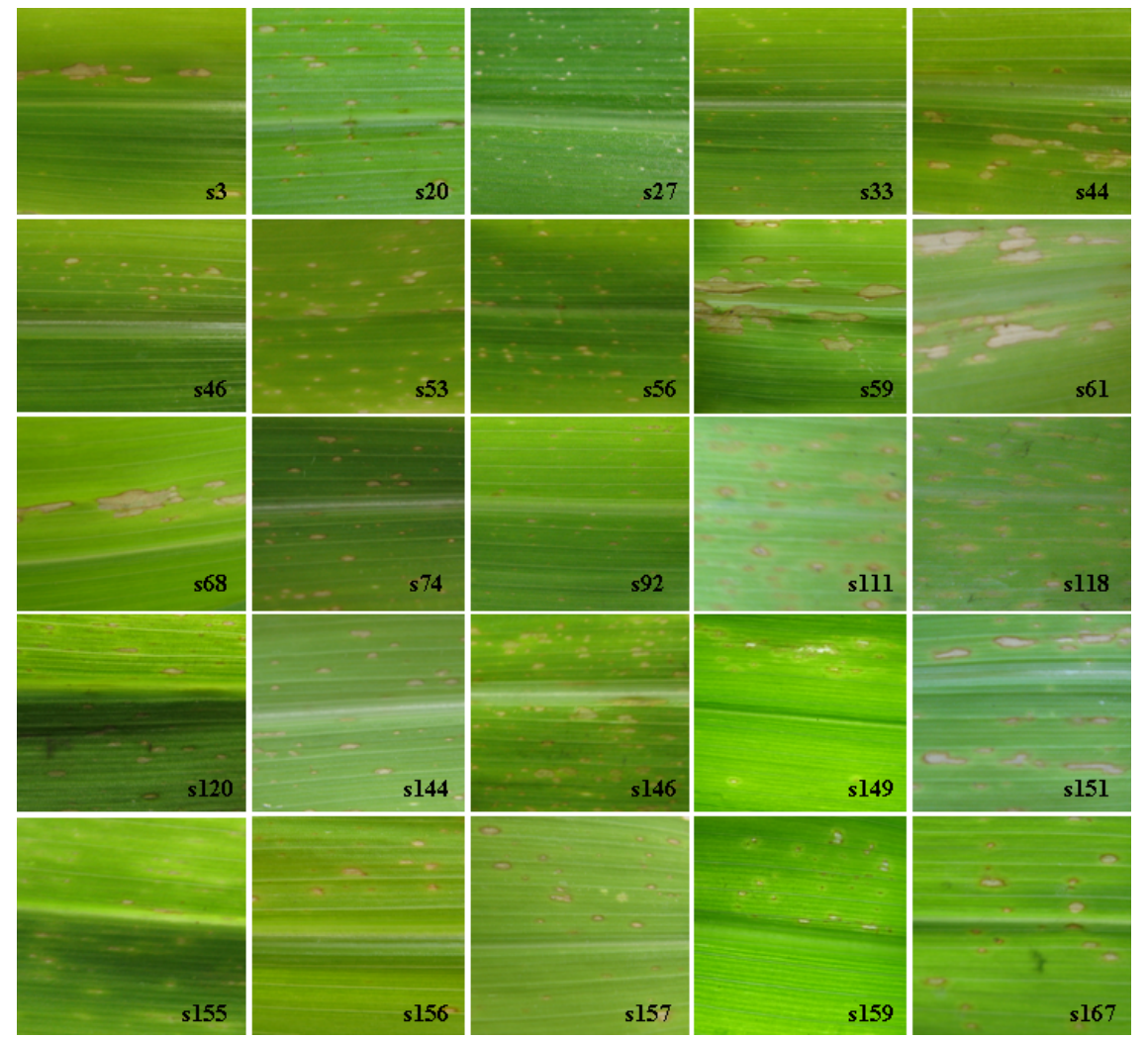

Figure 2: Symptoms of Cochliobolus carbonum isolates on corn inbred line MU-6. The isolates number is shown at the bottom right corner.

\begin{tabular}{|c|c|c|c|c|c|c|c|c|c|}
\hline \multirow[t]{2}{*}{ Isolates } & \multicolumn{8}{|c|}{ Corn inbred lines } & \multirow[t]{2}{*}{ Race } \\
\hline & MU-6 & W-0809 & MU-4 & SU-3-X & S37 & W-8053 & SY-2134 & SY-10-1 & \\
\hline s3 & LS $^{*}$ & LS & SOL & LS & LS & ALCS & LS & SOL & 3 \\
\hline$s 20$ & OS & OS & NO & OS & SOL & OS & OS & OS & 2 \\
\hline$s 27$ & SOL & LS & SOL & SOL & SOL & SOL & $\mathrm{SOL}$ & $\mathrm{SOL}$ & 3 \\
\hline s33 & SOL & SOL & SOL & LS & SOL & SOL & SOL & SOL & 3 \\
\hline s44 & LS & LS & ALCS & LS & ALCS & ALCS & LS & NO & 3 \\
\hline s46 & OS & OS & OS & OS & OS & ALCS & OS & OS & 3 \\
\hline$s 53$ & OS & OS & ALCS & LS & OS & ALCS & OS & OS & 3 \\
\hline s56 & OS & OS & OS & OS & SOL & OS & OS & OS & 2 \\
\hline s59 & LS & LS & ALCS & OS & OS & OS & OS & OS & 3 \\
\hline$s 61$ & LS & LS & ALCS & os & OS & os & os & OS & 3 \\
\hline$s 68$ & LS & LS & ALCS & ALCS & ALCS & ALCS & ALCS & ALCS & 3 \\
\hline s74 & OS & OS & ALCS & OS & SOL & OS & OS & os & 2 \\
\hline s92 & ALCS & OS & ALCS & OS & SOL & OS & os & OS & 2 \\
\hline s111 & OS & OS & ALCS & LS & LS & ALCS & OS & OS & 3 \\
\hline s118 & OS & OS & ALCS & LS & LS & ALCS & OS & OS & 3 \\
\hline s120 & OS & OS & ALCS & LS & LS & ALCS & OS & OS & 3 \\
\hline s144 & ALCS & ALCS & ALCS & ALCS & SOL & ALCS & ALCS & ALCS & 2 \\
\hline s146 & OS & os & OS & OS & SOL & OS & OS & OS & 2 \\
\hline s149 & ALCS & ALCS & NO & ALCS & LS & ALCS & ALCS & ALCS & 3 \\
\hline s151 & LS & OS & OS & LS & LS & ALCS & OS & OS & 3 \\
\hline s155 & OS & OS & OS & OS & SOL & OS & OS & OS & 2 \\
\hline s156 & OS & OS & OS & OS & SOL & OS & OS & OS & 2 \\
\hline s157 & OS & OS & OS & OS & SOL & OS & OS & OS & 2 \\
\hline s159 & ALCS & ALCS & OS & LS & LS & ALCS & ALCS & ALCS & 3 \\
\hline s167 & OS & OS & SOL & OS & SOL & OS & $\mathrm{NO}$ & OS & 2 \\
\hline
\end{tabular}

*LS=linear lesions ( $3.5 \times 0.5 \mathrm{~mm}$ ) (Susceptible to race 3$)$; SOL= Small oval spots $(\leq 0.5 \times 0.5 \mathrm{~mm})$ (Resistant to race 2$)$; OS=Oval spots $(1.9 \times 0.7 \mathrm{~mm})($ Susceptible to race 2 and resistant to race 3$)$; ALCS=Short linear lesions (1.0 $0.2 \mathrm{~mm}$ ) (Resistant to race 3); NO=No lesions (Resistant to both races 2 and 3 ).

Table 2: Race identification of 25 isolates of Cochliobolus carbonum on eight corn inbred lines. 
Citation: Zhang JX, Yi-Xin Wu, Hon-Hing Ho, Can-Hua Lu, Yue-Qiu He (2013) Cultural Characteristics and Pathogenic Variations among Cochliobolus carbonum Isolates in Yunnan Province of China. J Plant Pathol Microb 4: 210. doi:10.4172/2157-7471.1000210

\begin{tabular}{|c|c|c|c|c|c|c|c|c|c|c|c|c|c|}
\hline \multirow[t]{2}{*}{ Cultivars } & \multicolumn{13}{|c|}{ Isolates } \\
\hline & s3 & s20 & s27 & s33 & s44 & s46 & s53 & s56 & s59 & $s 61$ & s68 & s74 & s92 \\
\hline AN-4 & $6.1 \pm$ & $3 \pm$ & $5.8 \pm$ & $3 \pm$ & $5 \pm$ & $.5 \pm$ & $3 \pm$ & $.7 \pm 1.3 \mathrm{~S}$ & $5.8 \pm$ & $1.2 \pm$ & $1.0 \pm$ & $1.0 \pm$ & $4.6 \pm$ \\
\hline Y-68 & $5.8 \pm 1.7 \mathrm{~S}$ & $\pm 0.0 \mathrm{R}$ & $5 \pm 1.2 \mathrm{~S}$ & $7 \pm 0.2 \mathrm{~S}$ & $6 \pm 0.4 \mathrm{~S}$ & $3.4 \pm 0.4 \mathrm{~S}$ & $8 \pm 0.6 \mathrm{~S}$ & $4 \pm 1.5 \mathrm{~S}$ & $.7 \pm 0.7 \mathrm{~S}$ & $5.6 \pm 0.7 \mathrm{~S}$ & $4.3 \pm 1.1 \mathrm{~S}$ & $2.3 \pm 1.9 \mathrm{~S}$ & $3.6 \pm 0.9 \mathrm{~S}$ \\
\hline $\mathrm{YH}-1$ & $4.6 \pm 1.2 \mathrm{~S}$ & $5 \pm 1.0 \mathrm{~S}$ & $6 \pm 0.4 \mathrm{~S}$ & $8.1 \pm 0.2 \mathrm{~S}$ & $.8 \pm 0.2 S$ & $.4 \pm 0.9 \mathrm{~S}$ & $.7 \pm 0.8 \mathrm{~S}$ & $3.3 \pm 1.2 \mathrm{~S}$ & $6.5 \pm 0.8 \mathrm{~S}$ & $1.3 \pm 0.5 \mathrm{R}$ & $1.3 \pm 0.7 R$ & $1.0 \pm 0.0 R$ & $1.2 \pm 0.3 R$ \\
\hline QK-973 & $5.5 \pm 1.1 \mathrm{~S}$ & $2.3 \pm 0.9 \mathrm{~S}$ & $7 \pm 1.3 \mathrm{~S}$ & $9 \pm 0.3 \mathrm{R}$ & $7.3 \pm 0.6 \mathrm{~S}$ & $4.7 \pm 1.1 \mathrm{~S}$ & $4.9 \pm 0.9 \mathrm{~S}$ & $3.4 \pm 0.7 \mathrm{~S}$ & $5.4 \pm 1.2 \mathrm{~S}$ & $1.2 \pm 0.4 \mathrm{R}$ & $4.8 \pm 1.2 \mathrm{~S}$ & $1.0 \pm 0.0 \mathrm{R}$ & $4.3 \pm 1.3 \mathrm{~S}$ \\
\hline LH-25 & $6.6 \pm 1.3 \mathrm{~S}$ & $5 \pm 0.8 \mathrm{~S}$ & $4 \pm 0.2 \mathrm{~S}$ & $4 \pm 0.7 \mathrm{~S}$ & $5 \pm 0.5 \mathrm{~S}$ & $4.2 \pm 0.4 \mathrm{~S}$ & $1 \pm 0.7 \mathrm{~S}$ & $2.6 \pm 0.4 \mathrm{~S}$ & $6.1 \pm 1.3 \mathrm{~S}$ & $1.0 \pm 0.0 \mathrm{R}$ & $1.0 \pm 0.0 \mathrm{R}$ & $1.0 \pm 0.0 \mathrm{R}$ & $1.0 \pm 0.0 \mathrm{R}$ \\
\hline GY-10 & $4.9 \pm 1.4 \mathrm{~S}$ & $1.2 \pm 0.2 R$ & $5.8 \pm 1.3 \mathrm{~S}$ & $5 \pm 0.5 \mathrm{~S}$ & $6.5 \pm 0.3 \mathrm{~S}$ & $5.1 \pm 0.5 \mathrm{~S}$ & $5.4 \pm 0.6 \mathrm{~S}$ & $2.5 \pm 0.9 \mathrm{~S}$ & $6.2 \pm 0.7 \mathrm{~S}$ & $1.0 \pm 0.0 \mathrm{R}$ & $5.8 \pm 0.9 \mathrm{~S}$ & $2.5 \pm 0.7 \mathrm{~S}$ & $4.6 \pm 0.4 \mathrm{~S}$ \\
\hline BY-7 & $5.1 \pm 1.4 \mathrm{~S}$ & $2.3 \pm 0.4 \mathrm{~S}$ & $4.9 \pm 1.1 \mathrm{~S}$ & $5.4 \pm 0.7 \mathrm{~S}$ & $6.2 \pm 0.4 \mathrm{~S}$ & $5.3 \pm 0.8 \mathrm{~S}$ & $3.2 \pm 0.6 \mathrm{~S}$ & $2.7 \pm 0.8 \mathrm{~S}$ & $7.4 \pm 0.6 \mathrm{~S}$ & $1.0 \pm 0.0 \mathrm{R}$ & $2.3 \pm 0.3 \mathrm{R}$ & $1.0 \pm 0.0 \mathrm{R}$ & $3.4 \pm 0.5 \mathrm{~S}$ \\
\hline LY-26 & $6.2 \pm 1.9 \mathrm{~S}$ & $1.2 \pm 0.2 \mathrm{R}$ & $6.4 \pm 0.3 \mathrm{~S}$ & $8.3 \pm 0.3 \mathrm{~S}$ & $6.5 \pm 0.8 \mathrm{~S}$ & $5.7 \pm 0.3 \mathrm{~S}$ & $3.3 \pm 0.7 \mathrm{~S}$ & $3.1 \pm 0.7 \mathrm{~S}$ & $4.8 \pm 0.5 \mathrm{~S}$ & $5.1 \pm 0.6 \mathrm{~S}$ & $2.8 \pm 0.4 R$ & $1.1 \pm 0.5 R$ & $3.9 \pm 0.7 \mathrm{~S}$ \\
\hline SB-9 & $6.2 \pm 1.3 \mathrm{~S}$ & $0 \pm 0.0 \mathrm{R}$ & $5.2 \pm 0.5 \mathrm{~S}$ & $.3 \pm 0.7 \mathrm{~S}$ & $8.2 \pm 0.2 \mathrm{~S}$ & $4.6 \pm 0.6 \mathrm{~S}$ & $5.7 \pm 0.4 \mathrm{~S}$ & $2.9 \pm 0.8 \mathrm{~S}$ & $5.3 \pm 0.6 \mathrm{~S}$ & $6.3 \pm 0.3 \mathrm{~S}$ & $3.6 \pm 0.4 R$ & $1.0 \pm 0.0 \mathrm{~S}$ & $1.0 \pm 0.0 \mathrm{R}$ \\
\hline TY-67 & $7.2 \pm 1.5 \mathrm{~S}$ & $5 \pm 2.2 \mathrm{~S}$ & $9 \pm 0$ & $7 \pm 1.1 \mathrm{~S}$ & $6.7 \pm 0.3 \mathrm{~S}$ & $.3 \pm 0.8 \mathrm{~S}$ & $7 \pm 0.3 \mathrm{~S}$ & $1.0 \pm 0.0 \mathrm{R}$ & $6.2 \pm 0.9 \mathrm{~S}$ & $4.9 \pm 0.5 \mathrm{~S}$ & $4.4 \pm 1.2 \mathrm{~S}$ & $1.0 \pm 0.0 \mathrm{R}$ & $2.8 \pm 0.8 \mathrm{~S}$ \\
\hline S-70 & $5.3 \pm 1.6 \mathrm{~S}$ & $1.0 \pm 0.0 \mathrm{R}$ & $6.1 \pm 0.8 \mathrm{~S}$ & $1 \pm 0.3 \mathrm{~S}$ & $6 \pm 0.6 \mathrm{~S}$ & $4.7 \pm 0.5 \mathrm{~S}$ & $4.3 \pm 0.9 \mathrm{~S}$ & $3.4 \pm 1.3 \mathrm{~S}$ & $7.2 \pm 0.5 \mathrm{~S}$ & $1.1 \pm 0.3 \mathrm{R}$ & $1.2 \pm 0.3 R$ & $2.2 \pm 0.6 \mathrm{~S}$ & $2.6 \pm 0.6 \mathrm{~S}$ \\
\hline Q259 & $6.8 \pm 1.2 \mathrm{~S}$ & $1.0 \pm 0.0 \mathrm{R}$ & $2.8 \pm 0.5 R$ & $3.4 \pm 0.3 R$ & $5.9 \pm 0.3 \mathrm{~S}$ & $5.2 \pm 0.7 \mathrm{~S}$ & $3.7 \pm 0.7 \mathrm{~S}$ & $2.2 \pm 0.5 \mathrm{~S}$ & $2.6 \pm 0.5 \mathrm{R}$ & $1.1 \pm 0.5 \mathrm{R}$ & $1.3 \pm 0.4 \mathrm{R}$ & $1.0 \pm 0.0 \mathrm{R}$ & $3.7 \pm 0.8 \mathrm{~S}$ \\
\hline WY-1 & $4.8 \pm 1.5 \mathrm{~S}$ & $3.2 \pm 0.3 \mathrm{~S}$ & $7.7 \pm 0.8 \mathrm{~S}$ & $5.7 \pm 1.2 \mathrm{~S}$ & $7.2 \pm 0.4 \mathrm{~S}$ & $4.9 \pm 0.3 \mathrm{~S}$ & $4.6 \pm 0.6 \mathrm{~S}$ & $2.5 \pm 0.5 \mathrm{~S}$ & $4.3 \pm 1.1 \mathrm{~S}$ & $1.0 \pm 0.0 \mathrm{R}$ & $1.0 \pm 0.0 \mathrm{R}$ & $1.0 \pm 0.0 \mathrm{R}$ & $3.4 \pm 0.5 \mathrm{~S}$ \\
\hline XD-5 & $6.5 \pm 1.2 \mathrm{~S}$ & $1 \pm 0.2 \mathrm{R}$ & $6.3 \pm 0.6 \mathrm{~S}$ & $.8 \pm 0.3 \mathrm{~S}$ & $6.4 \pm 0.3 \mathrm{~S}$ & $4.3 \pm 0.6 \mathrm{~S}$ & $4.3 \pm 0$ & $2.3 \pm 0.3 \mathrm{~S}$ & $5.7 \pm 0.9 \mathrm{~S}$ & $1.2 \pm 0.3 \mathrm{R}$ & $5.4 \pm 0.8 \mathrm{~S}$ & $1.0 \pm 0.0 \mathrm{R}$ & $4.2 \pm 0.7 \mathrm{~S}$ \\
\hline $\mathrm{XH}-206$ & $5.8 \pm 0.9 \mathrm{~S}$ & $5 \pm 0.9 \mathrm{~s}$ & $6 \pm 1.2 \mathrm{~S}$ & $4 \pm 0.4 S$ & $3 \pm 0.4 \mathrm{~S}$ & $8 \pm 0.4 \mathrm{~S}$ & $7 \pm 0.6 \mathrm{~S}$ & $2 \pm 0.6 \mathrm{~S}$ & $6.2 \pm 0.4 \mathrm{~S}$ & $1.0 \pm 0.0 \mathrm{R}$ & $3.9 \pm 1.1 \mathrm{~S}$ & $2.4 \pm 0.8 \mathrm{~S}$ & $3.3 \pm 0.8 \mathrm{~S}$ \\
\hline CY-1 & 7 & $S$ & $S$ & S & $S$ & $\mathrm{~S}$ & $S$ & $7 \mathrm{~S}$ & $S$ & $S$ & 4.3 & $7 \mathrm{~S}$ & 25 \\
\hline LY-9 & $6.4 \pm 0.6 \mathrm{~S}$ & $2.7 \pm 0.1 \mathrm{~S}$ & $5.6 \pm 0.7 \mathrm{~S}$ & $7.3 \pm 0.3 \mathrm{~S}$ & $6.7 \pm 0.6 \mathrm{~S}$ & $5.6 \pm 0.4 \mathrm{~S}$ & $4.6 \pm 0.5 \mathrm{~S}$ & $3.8 \pm 0.8 \mathrm{~S}$ & $7.4 \pm 0.6 \mathrm{~S}$ & $5.6 \pm 0.7 \mathrm{~S}$ & $5.8 \pm 1.0 \mathrm{~S}$ & $2.8 \pm 0.6 \mathrm{~S}$ & $3.7 \pm 0.7 \mathrm{~S}$ \\
\hline ZJ-3 & $5.7 \pm 0.6 \mathrm{~S}$ & $1.1 \pm 0.3 R$ & $5.3 \pm 0.4 \mathrm{~S}$ & $3.5 \pm 1.2 \mathrm{R}$ & $6.9 \pm 0.5 \mathrm{~S}$ & $3.6 \pm 0.3 \mathrm{~S}$ & $5.5 \pm 0.8 \mathrm{~S}$ & $2.4 \pm 0.5 \mathrm{~S}$ & $7.8 \pm 0.3 \mathrm{~S}$ & $6.4 \pm 0.4 \mathrm{~S}$ & $4.2 \pm 1.2 \mathrm{~S}$ & $2.4 \pm 0.3 \mathrm{~S}$ & $1.8 \pm 0.4 \mathrm{~S}$ \\
\hline SF-169 & $7.3 \pm 0.8 \mathrm{~S}$ & $2.8 \pm 0.9 \mathrm{~S}$ & $4.8 \pm 0.8 \mathrm{~S}$ & $6.8 \pm 0.3 S$ & $7.3 \pm 0.6 \mathrm{~S}$ & $5.2 \pm 0.5 \mathrm{~S}$ & $5.6 \pm 0.6 \mathrm{~S}$ & $3.2 \pm 0.7 \mathrm{~S}$ & $5.7 \pm 0.4 \mathrm{~S}$ & $5.7 \pm 0.3 \mathrm{~S}$ & $1.0 \pm 0.0 \mathrm{R}$ & $2.7 \pm 0.5 \mathrm{~S}$ & $1.3 \pm 0.4 \mathrm{R}$ \\
\hline CL-98 & $5.3 \pm$ & $3.3 \pm 0$ & $5.8 \pm$ & $7 \pm$ & $6.1 \pm c$ & $3.3 \pm$ & $4.4 \pm$ & $3.3 \pm 0.9 \mathrm{~s}$ & $5.2 \pm$ & $6.3 \pm$ & $1,0 \pm$ & $3.1 \pm$ & $2.5 \pm 1.1 \mathrm{~S}$ \\
\hline $\mathrm{CX}$ & $S$ & $2.6 \pm$ & $5.3 \pm 1.3 \mathrm{~S}$ & S & S & S & S & $6 \mathrm{~S}$ & $\mathrm{~S}$ & $\mathrm{~S}$ & S & $\mathrm{S}$ & $7 \mathrm{~S}$ \\
\hline $\mathrm{ZH}-8$ & $6.4 \pm$ & $2.2 \pm 0$ & S & $S$ & S & $S$ & S & $3.3 \pm$ & S & $S$ & $0.6 \mathrm{~S}$ & $3 \mathrm{~S}$ & $1.2 \mathrm{~S}$ \\
\hline JY-2 & $5.9 \pm 0.6 \mathrm{~S}$ & $1.0 \pm 0.0 \mathrm{R}$ & $4.7 \pm 0.7 \mathrm{~S}$ & $5.5 \pm 0.4 \mathrm{~S}$ & $6.4 \pm 0.6 \mathrm{~S}$ & $4.9 \pm 0.4 \mathrm{~S}$ & $3.5 \pm 1.3 \mathrm{~S}$ & $2.4 \pm 0.5 \mathrm{~S}$ & $5.7 \pm 0.6 \mathrm{~S}$ & $6.6 \pm 0.5 \mathrm{~S}$ & $3.7 \pm 0.8 \mathrm{~S}$ & $2.7 \pm 0.6 \mathrm{~S}$ & $4.3 \pm 1.5 \mathrm{~S}$ \\
\hline DY-5 & $5.7 \pm 0.4 \mathrm{~S}$ & $1.0 \pm 0.0 \mathrm{R}$ & $6.9 \pm 0.4 \mathrm{~S}$ & $6.3 \pm 0.5 \mathrm{~S}$ & $7.6 \pm 0.3 \mathrm{~S}$ & $5.0 \pm 0.0 \mathrm{~S}$ & $5.3 \pm 1.1 \mathrm{~S}$ & $2.5 \pm 0.5 \mathrm{~S}$ & $5.7 \pm 0.3 \mathrm{~S}$ & $7.2 \pm 0.7 \mathrm{~S}$ & $3.5 \pm 0.7 \mathrm{~S}$ & $1.0 \pm 0.0 \mathrm{R}$ & $3.4 \pm 0.6 \mathrm{~S}$ \\
\hline SQ-1 & $6.9 \pm 0.2 \mathrm{~S}$ & $3.3 \pm 1.1 \mathrm{~S}$ & $5.3 \pm 0.6 \mathrm{~S}$ & $6.2 \pm 0.7 \mathrm{~S}$ & $7.1 \pm 0.2 \mathrm{~S}$ & $2.2 \pm 0.3 R$ & $6.2 \pm 0.9 \mathrm{~S}$ & $3.8 \pm 0.6 \mathrm{~S}$ & $6.3 \pm 0.7 \mathrm{~S}$ & $5.7 \pm 0.9 \mathrm{~S}$ & $3.5 \pm 0.5 \mathrm{~S}$ & $3.2 \pm 0.8 \mathrm{~S}$ & $2.6 \pm 1.1 \mathrm{~S}$ \\
\hline$X Y-696$ & $8.4 \pm 0.4 \mathrm{~S}$ & $2.6 \pm 0.7 \mathrm{~S}$ & $5.4 \pm 0.7 \mathrm{~S}$ & $7.6 \pm 0.4 \mathrm{~S}$ & $5.6 \pm 0.6 \mathrm{~S}$ & $4.6 \pm$ & $5.3 \pm 1.3 \mathrm{~S}$ & $4.5 \pm 0.9 \mathrm{~S}$ & $6.7 \pm 0.8 \mathrm{~S}$ & $4.6 \pm 0.5 \mathrm{~S}$ & $4.5 \pm 0.8 \mathrm{~S}$ & $2.3 \pm 1.3 \mathrm{~S}$ & $2.9 \pm 0.9 \mathrm{~S}$ \\
\hline ZD-2 & 6 & $S$ & a & $\mathrm{R}$ & S & $S$ & S & S & $S$ & $\mathrm{~S}$ & $R$ & $S$ & $3.4 \pm 1.3 \mathrm{~S}$ \\
\hline GHD-9 & $7.4 \pm 0.2 \mathrm{~S}$ & $3.2 \pm 1.1 \mathrm{~S}$ & $2.7 \pm 0.6 \mathrm{R}$ & $6.2 \pm 0.7 \mathrm{~S}$ & $7.4 \pm 0.3 \mathrm{~S}$ & $4.7 \pm 0.7 \mathrm{~S}$ & $4.7 \pm 0$ & $3.4 \pm 0.5 \mathrm{~S}$ & $5.3 \pm 0.7 \mathrm{~S}$ & $4.8 \pm 0.3 \mathrm{~S}$ & $4.9 \pm 0.8 \mathrm{~S}$ & $1.0 \pm 0.0 \mathrm{R}$ & $3.7 \pm 0.8 \mathrm{~S}$ \\
\hline WG-11 & $5.7 \pm 1.1 \mathrm{~S}$ & $2.5 \pm 0.8 \mathrm{~S}$ & $5.3 \pm 0.8 \mathrm{~S}$ & $2.6 \pm 0.4 R$ & $7.9 \pm 0.7 \mathrm{~S}$ & $4.9 \pm 0.5 \mathrm{~S}$ & $4.8 \pm 0.8 \mathrm{~S}$ & $3.6 \pm 0.6 \mathrm{~S}$ & $5.5 \pm 0.6 \mathrm{~S}$ & $4.9 \pm 0.8 \mathrm{~S}$ & $5.7 \pm 0.6 \mathrm{~S}$ & $1.0 \pm 0.0 \mathrm{R}$ & $3.2 \pm 0.5 \mathrm{~S}$ \\
\hline MZ-63 & $8.3 \pm 0.35$ & $2.6 \pm 1.0 \mathrm{~S}$ & $5.7 \pm 1.3 \mathrm{~S}$ & $5.8 \pm 0.3 \mathrm{~S}$ & $6.3 \pm 0.8 \mathrm{~S}$ & $3.7 \pm 0.6 \mathrm{~S}$ & $4.3 \pm 1.3 \mathrm{~S}$ & $3.3 \pm 0.8 \mathrm{~S}$ & $6.3 \pm 0.4 \mathrm{~S}$ & $5.0 \pm 0.7 \mathrm{~S}$ & $1.0 \pm 0.0 \mathrm{R}$ & $2.7 \pm 1.3 \mathrm{~S}$ & $3.5 \pm 1.3 \mathrm{~S}$ \\
\hline SY-1 & $6.4 \pm 1.0 \mathrm{~S}$ & $2.5 \pm 0.5 \mathrm{~S}$ & $4.4 \pm 1.4 \mathrm{~S}$ & $6.4 \pm 0.7 \mathrm{~S}$ & $7.1 \pm 0.5 \mathrm{~S}$ & $4.2 \pm 0.7 \mathrm{~S}$ & $4.2 \pm 0.4 \mathrm{~S}$ & $2.4 \pm 0.4 \mathrm{~S}$ & $6.7 \pm 0.5 \mathrm{~S}$ & $6.8 \pm 0.5 \mathrm{~S}$ & $1.0 \pm 0.0 \mathrm{R}$ & $2.2 \pm 1.5 \mathrm{~S}$ & $3.3 \pm 1.4 \mathrm{~S}$ \\
\hline GY-8 & $6.1 \pm 0.9 \mathrm{~S}$ & $3.2 \pm 0.6 \mathrm{R}$ & $4.7 \pm 0.6 \mathrm{~S}$ & $6.8 \pm 0.6 \mathrm{~S}$ & $6.3 \pm 0.7 \mathrm{~S}$ & $4.6 \pm 0.3 \mathrm{~S}$ & $5.7 \pm 0.5 \mathrm{~S}$ & $2.6 \pm 0.7 \mathrm{~S}$ & $6.9 \pm 0.7 \mathrm{~S}$ & $7.4 \pm 0.4 \mathrm{~S}$ & $4.6 \pm 1.5 \mathrm{~S}$ & $2.4 \pm 1.7 \mathrm{~S}$ & $2.7 \pm 1.5 \mathrm{~S}$ \\
\hline YD-1 & $4.8 \pm 1.1 \mathrm{~S}$ & $2.3 \pm 0.8 R$ & $4.1 \pm 0.7 \mathrm{~S}$ & $6.3 \pm 0.4 \mathrm{~S}$ & $6.7 \pm 0.4 \mathrm{~S}$ & $4.6 \pm 0.6 \mathrm{~S}$ & $4.6 \pm 0.7 \mathrm{~S}$ & $1.0 \pm 0.6 \mathrm{R}$ & $6.1 \pm 0.5 \mathrm{~S}$ & $6.6 \pm 0.7 \mathrm{~S}$ & $1.4 \pm 0.4 \mathrm{R}$ & $2.3 \pm 1.4 \mathrm{~S}$ & $3.8 \pm 1.3 \mathrm{~S}$ \\
\hline LD-99 & $5.4 \pm 0.5 \mathrm{~S}$ & $1.0 \pm 0.0 \mathrm{R}$ & $5.5 \pm 0.4 \mathrm{~S}$ & $2.5 \pm 0.2 \mathrm{R}$ & $6.4 \pm 0.5 \mathrm{~S}$ & $5.3 \pm 0.3 \mathrm{~S}$ & $5.2 \pm 0.8 \mathrm{~S}$ & $1.0 \pm 0.8 \mathrm{R}$ & $5.3 \pm 0.8 \mathrm{~S}$ & $5.3 \pm 0.7 \mathrm{~S}$ & $3.6 \pm 0.6 \mathrm{~S}$ & $2.5 \pm 1.5 \mathrm{~S}$ & $2.9 \pm 1.7 \mathrm{~S}$ \\
\hline LX-4 & $5.5 \pm 0.6 \mathrm{~S}$ & $3.2 \pm 0.7 \mathrm{~S}$ & $6.3 \pm 0.4 \mathrm{~S}$ & $5.9 \pm 0.9 \mathrm{~S}$ & $7.2 \pm 0.6 \mathrm{~S}$ & $3.2 \pm 0.8 \mathrm{~S}$ & $3.5 \pm 0.5 \mathrm{~S}$ & $2.5 \pm 0.6 \mathrm{~S}$ & $5.7 \pm 0.4 \mathrm{~S}$ & $5.2 \pm 0.8 \mathrm{~S}$ & $1.2 \pm 0.5 \mathrm{R}$ & $1.0 \pm 0.0 \mathrm{R}$ & $2.4 \pm 1.5 \mathrm{~S}$ \\
\hline TC-2 & $6.7 \pm 0.3 \mathrm{~S}$ & $1.0 \pm 0.0 \mathrm{R}$ & $6.1 \pm 0.7 \mathrm{~S}$ & $6.2 \pm 1.1 \mathrm{~S}$ & $6.3 \pm 0.7 \mathrm{~S}$ & $5.8 \pm 0.4 \mathrm{~S}$ & $3.8 \pm 0.7 \mathrm{~S}$ & $3.3 \pm 0.8 \mathrm{~S}$ & $7.1 \pm 0.7 \mathrm{~S}$ & $5.7 \pm 0.7 \mathrm{~S}$ & $1.0 \pm 0.0 \mathrm{R}$ & $1.0 \pm 0.0 \mathrm{R}$ & $2.6 \pm 1.5 \mathrm{~S}$ \\
\hline HY-92 & $7.6 \pm 0.4 \mathrm{~S}$ & $1.0 \pm 0.0 \mathrm{R}$ & $2.4 \pm 0.2 \mathrm{R}$ & $3.5 \pm 0.5 R$ & $6.2 \pm 0.3 \mathrm{~S}$ & $4.3 \pm 1.1 \mathrm{~S}$ & $4.4 \pm 0.5 \mathrm{~S}$ & $3.6 \pm 1.3 \mathrm{~S}$ & $6.2 \pm 0.4 \mathrm{~S}$ & $7.1 \pm 0.7 \mathrm{~S}$ & $4.7 \pm 0.7 \mathrm{~S}$ & $1.0 \pm 0.0 \mathrm{R}$ & $2.5 \pm 1.3 \mathrm{~S}$ \\
\hline DK-007 & $6.4 \pm 0.3 \mathrm{~S}$ & $1.0 \pm 0.0 \mathrm{R}$ & $2.8 \pm 0.5 R$ & $5.6 \pm 0.7 \mathrm{~S}$ & $7.4 \pm 0.5 \mathrm{~S}$ & $3.9 \pm 0.4 \mathrm{~S}$ & $4.2 \pm 0.6 \mathrm{~S}$ & $3.1 \pm 1.4 \mathrm{~S}$ & $6.3 \pm 0.5 \mathrm{~S}$ & $6.3 \pm 0.6 \mathrm{~S}$ & $1.4 \pm 0.9 R$ & $1.0 \pm 0.0 \mathrm{R}$ & $2.7 \pm 1.2 \mathrm{~S}$ \\
\hline HD-4 & $6.9 \pm 0.5 \mathrm{~S}$ & $2.3 \pm 0.6 \mathrm{~S}$ & $5.6 \pm 1.1 \mathrm{~S}$ & $6.3 \pm 0.8 \mathrm{~S}$ & $7.7 \pm 0.8 \mathrm{~S}$ & $3.2 \pm 0.6 \mathrm{~S}$ & $5.7 \pm 0.8 \mathrm{~S}$ & $2.9 \pm 1.5 \mathrm{~S}$ & $5.6 \pm 0.3 \mathrm{~S}$ & $5.5 \pm 0.4 \mathrm{~S}$ & $5.2 \pm 0.8 \mathrm{~S}$ & $2.1 \pm 1.7 \mathrm{~S}$ & $2.3 \pm 1.6 \mathrm{R}$ \\
\hline HD-5 & $6.6 \pm 0.5 \mathrm{~S}$ & $2.6 \pm 0.8 \mathrm{~S}$ & $4.5 \pm 0.7 \mathrm{~S}$ & $6.7 \pm 0.4 \mathrm{~S}$ & $8.0 \pm 0.6 \mathrm{~S}$ & $4.3 \pm 1.2 \mathrm{~S}$ & $4.3 \pm 0.5 \mathrm{~S}$ & $2.6 \pm 0.8 \mathrm{~S}$ & $5.2 \pm 1.1 \mathrm{~S}$ & $5.2 \pm 0.7 \mathrm{~S}$ & $4.5 \pm 0.5 \mathrm{~S}$ & $2.2 \pm 1.5 \mathrm{~S}$ & $2.4 \pm 1.8 \mathrm{~S}$ \\
\hline
\end{tabular}

$\mathrm{R}=$ Resistant, $\mathrm{S}=$ Susceptible

aDisease severity was recorded on a $1-9$ scale on the fourth and fifth inoculated leaves. $1=0 \% ; 2=1-10 \% ; 3=11-20 \% ; 4=21-30 \% ; 5=31-40 \% ; 6=41-50 \% ; 7=51-65 \% ; 8=65-$ $80 \% ; 9=>80 \%$ leaf area covered with lesions.

bMean and standard deviations were calculated from 10 plants.

Table 3: Pathogenicity and virulence (disease severity on a 1-9 scales) of 25 isolates of Cochliobolus carbonum on 40 corn cultivars.

$\mathrm{ml}$ sterile distilled water (SDW) and filtering the suspension through two layer of cheesecloth. The concentration of the spore suspension was determined with the aid of a hemocytometer and the suspension was diluted with SDW to obtain a concentration of $5 \times 10^{5}$ conidia $/ \mathrm{ml}$. A 5-6 leaf-stage corn plant was sprayed with the spore suspension containing $2 \mu \mathrm{l} / \mathrm{l}$ of polyoxyethylene sorbitan monolaureate 20 (Tween 20 ). Control plants were treated similarly with plain SDW. The inoculated plants were incubated overnight at $25^{\circ} \mathrm{C}$ in a dark moist chamber with $100 \%$ relative humidity and then returned to the greenhouse. Lesion types were recorded 6 days after inoculation, and the identification of races followed the method developed by Stanković et al. [18].

\section{Pathogenicity test}

Pathogenic variability of the isolates was evaluated in a greenhouse by testing all 25 isolates on 40 differential corn cultivars. The plants were spray-inoculated as described above. Plants were scored for disease reaction and disease severity 10 days after inoculation. Disease reaction was recorded as $\mathrm{R}=$ resistant (no lesions, small oval or short linear lesions); and $\mathrm{S}=$ susceptible (linear or oval lesions). Disease severity (Isolate virulence) was recorded on a 1-9 scale on the fourth and fifth inoculated leaves: $1=0 \% ; 2=1-10 \% ; 3=11-20 \% ; 4=21-30 \%$; $5=31-40 \% ; 6=41-50 \% ; 7=51-65 \% ; 8=65-80 \% ; 9=>80 \%$ leaf area covered with lesions. The experiment was conducted with 10 plants per test isolate, and it was repeated once.

\section{Results}

\section{Cultural characteristics}

The isolates of Cochliobolus sp. which were obtained from the lesions on corn leaves in the field were identified as C. carbonum based 
Citation: Zhang JX, Yi-Xin Wu, Hon-Hing Ho, Can-Hua Lu, Yue-Qiu He (2013) Cultural Characteristics and Pathogenic Variations among Cochliobolus carbonum Isolates in Yunnan Province of China. J Plant Pathol Microb 4: 210. doi:10.4172/2157-7471.1000210

primarily on the size and shape of conidia $[15,16]$. The isolates of $C$. carbonum showed differences in cultural characteristics (Table 1 and Figure 1). After 7 days of incubation various isolates produced grayishwhite to greyish-green hyphae on PLA whereas the reverse of the colonies was reddish violet to greyish-green. Conidia were mostly 3-9 septate and variable in shape, straight or moderately curved, dark or olivaceous brown, and the cells on the ends sometimes appeared paler than those in the middle, and measured 47.44-103.77×9.39-16.66 $\mu \mathrm{m}$ (av. $66.43 \times 12.70 \mu \mathrm{m}$ ). All isolates of $C$. carbonum produced abundant conidia on PLA medium (number of conidia $>10^{5} / 6 \mathrm{~mm}$ agar plate).

\section{Race identification}

Races 2 and 3 of C. carbonum were identified among the 25 isolates tested. Isolates of race 3 accounted for $60 \%$ of the isolates tested and predominated in all the regions surveyed (Table 2 and Figure 2).

\section{Pathogenicity variability}

The variable pathogenicity of $C$. carbonum isolates to corn leaves was summarized in Table 3 . The reaction of 40 corn cultivars indicated that most cultivars showed susceptibility to most of the 25 isolates of C. carbonum. Isolates S53, s146 and s151 were pathogenic to all corn cultivars, whereas LY-9, CX-6, XY-696 and HD-5 of corn cultivars showed susceptible response to all isolates. The reaction of 8 corn inbred lines to the 25 isolates indicated that MU- 4 and W- 8053 showed resistance response to all race 3 isolates, and s37 was resistant to all race 2 isolates. The remaining cultivars showed considerable variation in disease reactions. The virulence of $C$. carbonum isolate towards all the test cultivars exhibited considerable variability. The highly virulent isolates were s3, s27, s33, s44, s59, s118, s146, s151 and s167, and they belonged to race 3 except for s146 and s167; the less virulent isolates

\begin{tabular}{|c|c|c|c|c|c|c|c|c|c|c|c|c|}
\hline \multirow[t]{2}{*}{ Cultivars } & \multicolumn{12}{|l|}{ Isolates } \\
\hline & s111 & s118 & s120 & s144 & s146 & s149 & s151 & s155 & s156 & s157 & s159 & s167 \\
\hline AN-4 & $5.7 \pm 0.8 \mathrm{~S}^{*}$ & $7.3 \pm 0.6^{\mathrm{a}, \mathrm{b}} \mathrm{S}$ & $1.0 \pm 0.0 \mathrm{R}$ & $2.3 \pm 0.3 \mathrm{~S}$ & $7.6 \pm 0.4 \mathrm{~S}$ & $3.8 \pm 0.7 \mathrm{~S}$ & $7.4 \pm 0.3 \mathrm{~S}$ & $3.8 \pm 0.6 \mathrm{~S}$ & $2.8 \pm 0.7 \mathrm{~S}$ & $3.4 \pm 0.5 \mathrm{~S}$ & $4.6 \pm 0.4 \mathrm{~S}$ & $7.4 \pm 0.5 \mathrm{~S}$ \\
\hline YY-68 & $5.6 \pm 0.6 \mathrm{~S}$ & $6.7 \pm 0.4 \mathrm{~S}$ & $4.5 \pm 0.8 \mathrm{~S}$ & $2.6 \pm 0.5 \mathrm{~S}$ & $5.6 \pm 0.7 \mathrm{~S}$ & $4.5 \pm 0.5 \mathrm{~S}$ & $5.8 \pm 0.4 \mathrm{~S}$ & $4.5 \pm 0.7 \mathrm{~S}$ & $3.9 \pm 0.6 \mathrm{~S}$ & $4.3 \pm 0.7 \mathrm{~S}$ & $5.3 \pm 0.6 \mathrm{~S}$ & $6.4 \pm 0.7 \mathrm{~S}$ \\
\hline $\mathrm{YH}-1$ & $6.3 \pm 1.6 \mathrm{~S}$ & $7.5 \pm 0.5 \mathrm{~S}$ & $3.8 \pm 0.6 \mathrm{~S}$ & $3.5 \pm 0.6 \mathrm{~S}$ & $6.3 \pm 0.9 \mathrm{~S}$ & $4.2 \pm 0.7 \mathrm{~S}$ & $6.5 \pm 0.5 \mathrm{~S}$ & $3.5 \pm 0.5 \mathrm{~S}$ & $3.3 \pm 0.3 \mathrm{~S}$ & $2.8 \pm 1.3 \mathrm{~S}$ & $5.9 \pm 0.7 \mathrm{~S}$ & $6.7 \pm 0.7 \mathrm{~S}$ \\
\hline QK-973 & $4.7 \pm 1.1 \mathrm{~S}$ & $6.4 \pm 0.3 \mathrm{~S}$ & $2.3 \pm 0.4 \mathrm{R}$ & $3.2 \pm 0.5 \mathrm{~S}$ & $5.4 \pm 1.7 \mathrm{~S}$ & $4.7 \pm 0.9 \mathrm{~S}$ & $4.6 \pm 0.7 \mathrm{~S}$ & $1.6 \pm 0.4 R$ & $1.0 \pm 0.0 \mathrm{R}$ & $2.5 \pm 0.8 \mathrm{~S}$ & $6.2 \pm 0.3 \mathrm{~s}$ & $7.4 \pm 0.8 \mathrm{~S}$ \\
\hline LH-25 & $7.2 \pm 0.5 \mathrm{~S}$ & $5.7 \pm 0.5 \mathrm{~S}$ & $2.6 \pm 0.5 R$ & $3.8 \pm 0.8 \mathrm{~S}$ & $5.7 \pm 0.4 \mathrm{~S}$ & $3.3 \pm 0.4 \mathrm{~S}$ & $7.6 \pm 0.6 \mathrm{~S}$ & $1.0 \pm 0.0 \mathrm{R}$ & $4.1 \pm 0.7 \mathrm{~S}$ & $2.8 \pm 0.5 \mathrm{~S}$ & $3.8 \pm 0.6 \mathrm{~S}$ & $5.7 \pm 0.4 \mathrm{~S}$ \\
\hline GY-10 & $6.3 \pm 0.6 \mathrm{~S}$ & $5.9 \pm 0.7 \mathrm{~S}$ & $4.5 \pm 0.4 \mathrm{~S}$ & $2.3 \pm 0.2 \mathrm{~S}$ & $6.5 \pm 0.7 \mathrm{~S}$ & $4.6 \pm 0.5 \mathrm{~S}$ & $5.4 \pm 0.5 \mathrm{~S}$ & $3.7 \pm 0.7 \mathrm{~S}$ & $4.6 \pm 0.9 \mathrm{~S}$ & $3.5 \pm 0.4 \mathrm{~S}$ & $7.4 \pm 0.8 \mathrm{~S}$ & $5.3 \pm 0.6 \mathrm{~S}$ \\
\hline BY-7 & $5.4 \pm 0.9 \mathrm{~S}$ & $2.9 \pm 1.3 \mathrm{R}$ & $4.8 \pm 0.6 \mathrm{~S}$ & $2.6 \pm 0.3 \mathrm{~S}$ & $6.8 \pm 0.3 \mathrm{~S}$ & $5.5 \pm 0.7 \mathrm{~S}$ & $4.7 \pm 0.3 \mathrm{~S}$ & $4.5 \pm 0.9 \mathrm{~S}$ & $3.9 \pm 0.5 \mathrm{~S}$ & $3.6 \pm 0.6 \mathrm{~S}$ & $7.1 \pm 0.6 \mathrm{~S}$ & $6.2 \pm 0.3 \mathrm{~S}$ \\
\hline LY-26 & $6.4 \pm 1.2 \mathrm{~S}$ & $6.4 \pm 0.4 \mathrm{~S}$ & $4.8 \pm 0.3 \mathrm{~S}$ & $2.4 \pm 0.4 \mathrm{~S}$ & $5.3 \pm 0.6 \mathrm{~S}$ & $1.2 \pm 0.3 R$ & $5.3 \pm 0.6 \mathrm{~S}$ & $1.0 \pm 0.0 \mathrm{R}$ & $3.4 \pm 0.3 \mathrm{~S}$ & $3.9 \pm 0.7 \mathrm{~S}$ & $6.5 \pm 0.5 \mathrm{~S}$ & $6.4 \pm 0.6 \mathrm{~S}$ \\
\hline SB-9 & $5.2 \pm 0.3 \mathrm{~S}$ & $5.7 \pm 0.3 \mathrm{~S}$ & $4.1 \pm 0.5 \mathrm{~S}$ & $3.6 \pm 0.6 \mathrm{~S}$ & $5.7 \pm 0.4 \mathrm{~S}$ & $1.0 \pm 0.0 \mathrm{R}$ & $6.7 \pm 0.7 \mathrm{~S}$ & $1.0 \pm 0.0 \mathrm{R}$ & $3.3 \pm 0.6 \mathrm{~S}$ & $2.4 \pm 0.6 \mathrm{~S}$ & $5.4 \pm 0.3 \mathrm{~S}$ & $6.7 \pm 0.2 \mathrm{~S}$ \\
\hline TY-67 & $5.6 \pm 0.5 \mathrm{~S}$ & $5.4 \pm 0.6 \mathrm{~S}$ & $3.9 \pm 0.6 \mathrm{~S}$ & $3.6 \pm 0.5 \mathrm{~S}$ & $6.3 \pm 0.7 \mathrm{~S}$ & $1.0 \pm 0.0 \mathrm{R}$ & $5.9 \pm 0.5 \mathrm{~S}$ & $3.5 \pm 0.4 \mathrm{~S}$ & $3.7 \pm 0.5 \mathrm{~S}$ & $2.7 \pm 0.9 \mathrm{~S}$ & $5.9 \pm 0.2 \mathrm{~S}$ & $6.8 \pm 0.9 \mathrm{~S}$ \\
\hline S-70 & $6.1 \pm 0.7 \mathrm{~S}$ & $7.3 \pm 0.6 \mathrm{~S}$ & $3.7 \pm 0.3 \mathrm{R}$ & $3.5 \pm 0.7 \mathrm{~S}$ & $5.7 \pm 0.6 \mathrm{~S}$ & $3.7 \pm 0.5 \mathrm{~S}$ & $6.4 \pm 0.6 \mathrm{~S}$ & $4.8 \pm 0.6 \mathrm{~S}$ & $2.7 \pm 0.6 \mathrm{~S}$ & $2.8 \pm 0.4 \mathrm{~S}$ & $4.7 \pm 0.6 \mathrm{~S}$ & $7.2 \pm 0.5 \mathrm{~S}$ \\
\hline Q259 & $7.3 \pm 0.4 \mathrm{~S}$ & $6.3 \pm 0.7 \mathrm{~S}$ & $3.9 \pm 0.3 \mathrm{~S}$ & $2.1 \pm 0.6 \mathrm{~S}$ & $6.8 \pm 0.5 \mathrm{~S}$ & $3.2 \pm 0.6 \mathrm{~S}$ & $5.7 \pm 0.4 \mathrm{~S}$ & $2.5 \pm 0.7 \mathrm{~S}$ & $2.6 \pm 0.7 \mathrm{~S}$ & $2.7 \pm 0.6 \mathrm{~S}$ & $4.3 \pm 0.5 \mathrm{~S}$ & $5.9 \pm 0.7 \mathrm{~S}$ \\
\hline WY-1 & $2.6 \pm 0.3 \mathrm{R}$ & $5.5 \pm 0.5 \mathrm{~S}$ & $3.5 \pm 0.4 \mathrm{~S}$ & $3.4 \pm 0.7 \mathrm{~S}$ & $5.5 \pm 0.6 \mathrm{~S}$ & $4.6 \pm 0.4 \mathrm{~S}$ & $5.4 \pm 0.7 \mathrm{~S}$ & $3.8 \pm 0.5 \mathrm{~S}$ & $3.8 \pm 0.5 \mathrm{~S}$ & $3.6 \pm 0.3 \mathrm{~S}$ & $5.7 \pm 0.8 \mathrm{~S}$ & $6.4 \pm 0.5 \mathrm{~S}$ \\
\hline XD-5 & $5.4 \pm 0.9 \mathrm{~S}$ & $6.8 \pm 0.6 \mathrm{~S}$ & $3.6 \pm 0.5 \mathrm{~S}$ & $3.4 \pm 0.2 \mathrm{~S}$ & $5.5 \pm 0.5 \mathrm{~S}$ & $1.0 \pm 0.0 \mathrm{R}$ & $6.2 \pm 0.6 \mathrm{~S}$ & $2.8 \pm 0.7 \mathrm{~S}$ & $2.6 \pm 0.6 \mathrm{~S}$ & $3.4 \pm 0.6 \mathrm{~S}$ & $6.3 \pm 0.6 \mathrm{~S}$ & $7.3 \pm 0.7 \mathrm{~S}$ \\
\hline $\mathrm{XH}-206$ & $6.2 \pm 0.5 \mathrm{~S}$ & $6.5 \pm 0.7 \mathrm{~S}$ & $4.2 \pm 0.6 \mathrm{~S}$ & $2.9 \pm 0.3 \mathrm{~S}$ & $5.2 \pm 1.3 \mathrm{~S}$ & $6.2 \pm 0.3 \mathrm{~S}$ & $6.8 \pm 0.5 \mathrm{~S}$ & $3.3 \pm 0.5 \mathrm{~S}$ & $3.3 \pm 0.7 \mathrm{~S}$ & $3.3 \pm 0.5 \mathrm{~S}$ & $6.8 \pm 0.3 \mathrm{~S}$ & $7.8 \pm 0.9 \mathrm{~S}$ \\
\hline CY-1 & $1.8 \pm 0.6 \mathrm{R}$ & $5.2 \pm 0.5 \mathrm{~S}$ & $4.6 \pm 0.7 \mathrm{~S}$ & $2.2 \pm 0.4 \mathrm{~S}$ & $6.7 \pm 0.4 \mathrm{~S}$ & $5.6 \pm 0.5 \mathrm{~S}$ & $6.5 \pm 0.6 \mathrm{~S}$ & $1.0 \pm 0.0 \mathrm{R}$ & $2.9 \pm 0.5 \mathrm{~S}$ & $3.7 \pm 0.4 \mathrm{~S}$ & $6.3 \pm 0.7 \mathrm{~S}$ & $6.4 \pm 0.2 \mathrm{~S}$ \\
\hline LY-9 & $4.5 \pm 0.7 \mathrm{~S}$ & $6.4 \pm 0.6 \mathrm{~S}$ & $4.7 \pm 0$ & $2.5 \pm$ & $6.4 \pm$ & $5.2 \pm$ & 7.5 & $3.6 \pm$ & $2.8 \pm$ & $4.2 \pm$ & $5.7 \pm$ & $5.5 \pm$ \\
\hline ZJ-3 & $5.7 \pm 1.6 \mathrm{~S}$ & $6.7 \pm 0.7 \mathrm{~S}$ & $4.4 \pm 0.7 \mathrm{~S}$ & $2.7 \pm 0.6 \mathrm{~S}$ & $7.3 \pm 0.7 \mathrm{~S}$ & $4.8 \pm 0.5 \mathrm{~S}$ & $4.7 \pm 0.5 \mathrm{~S}$ & $3.7 \pm 0.7 \mathrm{~S}$ & $3.3 \pm 0.7 \mathrm{~S}$ & $3.6 \pm 0.9 \mathrm{~S}$ & $5.4 \pm 0.4 \mathrm{~S}$ & $5.7 \pm 0.6 \mathrm{~S}$ \\
\hline SF-169 & $4.2 \pm 0.9 \mathrm{~S}$ & $5.6 \pm 0.9 \mathrm{~S}$ & $3.6 \pm 0.8 \mathrm{~S}$ & $2.8 \pm 0.7 \mathrm{~S}$ & $5.6 \pm 0.7 \mathrm{~S}$ & $4.6 \pm 0.6 \mathrm{~S}$ & $4.8 \pm 0.8 \mathrm{~S}$ & $2.5 \pm 0.6 \mathrm{~S}$ & $2.8 \pm 0.6 \mathrm{~S}$ & $3.4 \pm 0.5 R$ & $5.9 \pm 0.7 \mathrm{~S}$ & $5.3 \pm 0.7 \mathrm{~S}$ \\
\hline CL-98 & $4.9 \pm 0.7 \mathrm{~S}$ & $5.2 \pm 0.5 \mathrm{~S}$ & $2.1 \pm 0.3 R$ & $2.4 \pm 0.6 \mathrm{~S}$ & $6.2 \pm 0.6 \mathrm{~S}$ & $6.3 \pm 0.7 \mathrm{~S}$ & $4.1 \pm 0.6 \mathrm{~S}$ & $3.6 \pm 0.7 \mathrm{~S}$ & $4.3 \pm 0.4 \mathrm{~S}$ & $2.8 \pm 0.6 \mathrm{~S}$ & $7.2 \pm 0.6 \mathrm{~S}$ & $5.7 \pm 0.5 \mathrm{~S}$ \\
\hline CX-6 & $4.9 \pm 0.8 \mathrm{~S}$ & $6.7 \pm 0.6 \mathrm{~S}$ & $3.3 \pm 0.7 \mathrm{~S}$ & $2.5 \pm 0.7 \mathrm{~S}$ & $6.7 \pm 0.4 \mathrm{~S}$ & $5.7 \pm 0.4 \mathrm{~S}$ & $7.2 \pm 0.4 \mathrm{~S}$ & $3.9 \pm 0.5 \mathrm{~S}$ & $3.6 \pm 0.5 \mathrm{~S}$ & $2.6 \pm 0.5 \mathrm{~S}$ & $7.3 \pm 0.3 \mathrm{~S}$ & $6.7 \pm 0.7 \mathrm{~S}$ \\
\hline $\mathrm{ZH}-8$ & $5.2 \pm 0.7 \mathrm{~S}$ & $2.2 \pm 0.7 \mathrm{R}$ & $3.6 \pm 0.5 \mathrm{~S}$ & $3.4 \pm 0.9 \mathrm{~S}$ & $5.5 \pm 0.4 \mathrm{~S}$ & $4.5 \pm 0.6 \mathrm{~S}$ & $4.8 \pm 0.6 \mathrm{~S}$ & $1.0 \pm 0.0 \mathrm{R}$ & $3.8 \pm 0.7 \mathrm{~S}$ & $3.8 \pm 0.4 \mathrm{~S}$ & $4.7 \pm 0.4 \mathrm{~S}$ & $6.4 \pm 0.9 \mathrm{~s}$ \\
\hline JY-2 & $4.6 \pm 0.6 \mathrm{~S}$ & $6.3 \pm$ & $3.7 \pm$ & 2.3 & 6.3 & $6 \mathrm{~S}$ & $\mathrm{~S}$ & S & $.5 \mathrm{~S}$ & 85 & S & $5.8 \pm 0.6 \mathrm{~S}$ \\
\hline DY-5 & $4.9 \pm 0.7 \mathrm{~S}$ & $6.4 \pm 0.4 \mathrm{~S}$ & $3.9 \pm 0.5 \mathrm{~S}$ & $2.6 \pm 0.7 \mathrm{~S}$ & $5.7 \pm 0.7 \mathrm{~S}$ & $4.8 \pm 0.4 \mathrm{~S}$ & $6.3 \pm 0.9 \mathrm{~S}$ & $2.9 \pm 0.5 \mathrm{~S}$ & $3.8 \pm 0.6 \mathrm{~S}$ & $3.7 \pm 0.3 \mathrm{~S}$ & $5.8 \pm 0.5 \mathrm{~S}$ & $7.8 \pm 0.5 \mathrm{~S}$ \\
\hline SQ-1 & $2.8 \pm 0.5 \mathrm{R}$ & $5.6 \pm 0.5 \mathrm{~S}$ & $4.2 \pm 0.6 \mathrm{~S}$ & $3.7 \pm 0.6 \mathrm{~S}$ & $5.3 \pm 0.7 \mathrm{~S}$ & $1.3 \pm 0.6 \mathrm{R}$ & $4.8 \pm 0.3 \mathrm{~S}$ & $2.5 \pm 0.7 \mathrm{~S}$ & $4.2 \pm 0.3 \mathrm{~S}$ & $3.3 \pm 0.2 \mathrm{~S}$ & $6.4 \pm 0.3 \mathrm{~S}$ & $5.4 \pm 0.4 \mathrm{~S}$ \\
\hline$X Y-696$ & $6.3 \pm 0.5 \mathrm{~S}$ & $5.7 \pm 0.6 \mathrm{~S}$ & $4.6 \pm 0.4 \mathrm{~S}$ & $3.2 \pm 0.5 \mathrm{~S}$ & $6.6 \pm 0.9 \mathrm{~S}$ & $6.3 \pm 0.5 \mathrm{~S}$ & $5.9 \pm 0.5 \mathrm{~S}$ & $3.5 \pm 0.9 \mathrm{~S}$ & $4.4 \pm 0.3 \mathrm{~S}$ & $3.6 \pm 0.8 \mathrm{~S}$ & $6.1 \pm 0.7 \mathrm{~S}$ & $5.7 \pm 0.7 \mathrm{~S}$ \\
\hline ZD-277 & $3.8 \pm 0.4 \mathrm{~S}$ & $5.3 \pm 0.4 \mathrm{~S}$ & $3.2 \pm 0.3 \mathrm{~S}$ & $2.8 \pm 0.4 \mathrm{~S}$ & $7.2 \pm 0.7 \mathrm{~S}$ & $5.4 \pm 0.8 \mathrm{~S}$ & $4.6 \pm 0.7 \mathrm{~S}$ & $1.0 \pm 0.0 \mathrm{R}$ & $3.2 \pm 0.9 \mathrm{~S}$ & $4.2 \pm 0.5 \mathrm{~S}$ & $5.7 \pm 0.4 \mathrm{~S}$ & $6.3 \pm 0.3 \mathrm{~s}$ \\
\hline GHD-9 & $5.4 \pm 0.3 S$ & $6.1 \pm 0.7 \mathrm{~S}$ & $3.4 \pm 0.4 \mathrm{~S}$ & $3.3 \pm 0.6 \mathrm{~S}$ & $5.4 \pm 0.5 \mathrm{~S}$ & $3.8 \pm 0.6 \mathrm{~S}$ & $4.5 \pm 0.4 \mathrm{~S}$ & $2.5 \pm 0.4 \mathrm{~S}$ & $2.7 \pm 0.4 \mathrm{~S}$ & $4.5 \pm 0.5 \mathrm{~S}$ & $5 \mathrm{~S}$ & $6.7 \pm 0.4 \mathrm{~S}$ \\
\hline WG-11 & $5.5 \pm 0.7 \mathrm{~S}$ & $5.8 \pm$ & $3.1 \pm c$ & $7 \mathrm{~S}$ & $S$ & $4.3=$ & $5.7 \pm$ & $0 \mathrm{R}$ & $0.4 \mathrm{~S}$ & $0.6 \mathrm{~S}$ & $0.5 \mathrm{~S}$ & $6.2 \pm 0.5 \mathrm{~S}$ \\
\hline MZ-63 & $4.1 \pm 0.5 \mathrm{~S}$ & $2.9 \pm 0.5 \mathrm{R}$ & $1.0 \pm 0.0 \mathrm{R}$ & $2.7 \pm 0.6 \mathrm{~S}$ & $7.8 \pm 0.7 \mathrm{~S}$ & $5.7 \pm 0.5 \mathrm{~S}$ & $5.8 \pm 0.7 \mathrm{~S}$ & $1.0 \pm 0.0 \mathrm{R}$ & $4.6 \pm 0.6 \mathrm{~S}$ & $3.7 \pm 0.7 \mathrm{~S}$ & $6.2 \pm 0.6 \mathrm{~S}$ & $7.3 \pm 0.2 \mathrm{~S}$ \\
\hline SY-1 & $4.6 \pm 0.3 \mathrm{~S}$ & $5.7 \pm 0.7 \mathrm{~S}$ & $2.3 \pm 0.0 \mathrm{~S}$ & $3.3 \pm 0.5 \mathrm{~S}$ & $6.4 \pm 0.3 \mathrm{~S}$ & $6.5 \pm 0.7 \mathrm{~S}$ & $7.8 \pm 0.5 \mathrm{~S}$ & $1.0 \pm 0.0 R$ & $3.7 \pm 0.7 \mathrm{~S}$ & $2.9 \pm 0.5 \mathrm{~S}$ & $7.3 \pm 0.4 \mathrm{~S}$ & $6.4 \pm 0.5 \mathrm{~S}$ \\
\hline GY-8 & $6.3 \pm 0.5 \mathrm{~S}$ & $2.4 \pm 0.6 \mathrm{R}$ & $3.2 \pm 0.5 \mathrm{~S}$ & $3.6 \pm 0.6 \mathrm{~S}$ & $5.7 \pm 0.5 \mathrm{~S}$ & $3.5 \pm 0.8 \mathrm{~S}$ & $5.4 \pm 0.6 \mathrm{~S}$ & $1.0 \pm 0.0 \mathrm{R}$ & $4.6 \pm 0.8 \mathrm{~S}$ & $4.3 \pm 0.7 \mathrm{~S}$ & $4.7 \pm 0.7 \mathrm{~S}$ & $5.7 \pm 0.7 \mathrm{~S}$ \\
\hline YD-1 & $5.8 \pm 0.3 S$ & $5.4 \pm 0.7 \mathrm{~S}$ & $4.3 \pm 0.6 \mathrm{~S}$ & $2.8 \pm 0.8 \mathrm{~S}$ & $4.7 \pm 0.5 \mathrm{~S}$ & $3.2 \pm 0.7 \mathrm{~S}$ & $6.8 \pm 0.4 \mathrm{~S}$ & $1.0 \pm 0.0 \mathrm{R}$ & $3.8 \pm 0.9 \mathrm{~S}$ & $2.3 \pm 0.5 \mathrm{~S}$ & $5.6 \pm 0.5 \mathrm{~S}$ & $5.8 \pm 0.4 \mathrm{~S}$ \\
\hline LD-99 & $6.3 \pm 0.3 \mathrm{~S}$ & $6.5 \pm 0.5 \mathrm{~S}$ & $2.5 \pm 0.4 \mathrm{~S}$ & $3.4 \pm 0.3 \mathrm{~S}$ & $6.3 \pm 0.6 \mathrm{~S}$ & $3.9 \pm 0.5 \mathrm{~S}$ & $5.3 \pm 0.6 \mathrm{~S}$ & $1.0 \pm 0.0 \mathrm{R}$ & $3.3 \pm 0.4 \mathrm{~S}$ & $3.8 \pm 0.7 \mathrm{~S}$ & $7.8 \pm 0.6 \mathrm{~S}$ & $6.4 \pm 0.8 \mathrm{~S}$ \\
\hline LX-4 & $4.7 \pm 0.9 \mathrm{~S}$ & $6.8 \pm 0.6 \mathrm{~S}$ & $3.4 \pm 0.6 \mathrm{~S}$ & $3.5 \pm 0.4 \mathrm{~S}$ & $6.5 \pm 0.4 \mathrm{~S}$ & $4.3 \pm 0.6 \mathrm{~S}$ & $6.4 \pm 0.7 \mathrm{~S}$ & $3.5 \pm 0.6 \mathrm{~S}$ & $1.0 \pm 0.0 \mathrm{R}$ & $3.6 \pm 0.3 \mathrm{~S}$ & $6.3 \pm 0.4 \mathrm{~S}$ & $6.8 \pm 0.5 \mathrm{~S}$ \\
\hline TC-2 & $7.2 \pm 0.4 \mathrm{~S}$ & $5.2 \pm 0.3 \mathrm{~S}$ & $1.8 \pm 0.4 R$ & $3.5 \pm 0.6 \mathrm{~S}$ & $7.2 \pm 0.7 \mathrm{~S}$ & $4.6 \pm 0.6 \mathrm{~S}$ & $6.3 \pm 0.5 \mathrm{~S}$ & $1.0 \pm 0.0 \mathrm{R}$ & $3.3 \pm 0.6 \mathrm{~S}$ & $3.4 \pm 0.6 \mathrm{~S}$ & $6.4 \pm 0.7 \mathrm{~S}$ & $6.1 \pm 0.6 \mathrm{~S}$ \\
\hline HY-92 & $5.4 \pm 0.6 \mathrm{~S}$ & $5.3 \pm 0.7 \mathrm{~S}$ & $3.2 \pm 0.7 \mathrm{~S}$ & $3.1 \pm 0.7 \mathrm{~S}$ & $5.9 \pm 0.9 \mathrm{~S}$ & $6.7 \pm 0.4 \mathrm{~S}$ & $5.8 \pm 0.3 \mathrm{~S}$ & $3.4 \pm 0.7 \mathrm{~S}$ & $4.3 \pm 0.4 \mathrm{~S}$ & $4.1 \pm 0.4 \mathrm{~S}$ & $6.8 \pm 0.5 \mathrm{~S}$ & $6.5 \pm 0.5 \mathrm{~S}$ \\
\hline DK-007 & $4.2 \pm 0.7 \mathrm{~S}$ & $5.8 \pm 0.5 \mathrm{~S}$ & $2.7 \pm 0.5 \mathrm{~S}$ & $3.8 \pm 0.5 \mathrm{~S}$ & $6.3 \pm 0.3 \mathrm{~S}$ & $5.5 \pm 0.6 \mathrm{~S}$ & $6.0 \pm 0.2 \mathrm{~S}$ & $2.8 \pm 0.5 \mathrm{~S}$ & $3.7 \pm 0.7 \mathrm{~S}$ & $3.9 \pm 0.4 \mathrm{~S}$ & $5.4 \pm 0.3 \mathrm{~S}$ & $6.7 \pm 0.7 \mathrm{~S}$ \\
\hline HD-4 & $4.3 \pm 0.5 \mathrm{~S}$ & $5.5 \pm 0.6 \mathrm{~S}$ & $2.3 \pm 0.4 \mathrm{~S}$ & $3.0 \pm 0.4 \mathrm{~S}$ & $6.8 \pm 0.4 \mathrm{~S}$ & $3.8 \pm 0.7 \mathrm{~S}$ & $5.7 \pm 0.6 \mathrm{~S}$ & $3.4 \pm 0.3 \mathrm{~S}$ & $3.6 \pm 0.5 \mathrm{~S}$ & $3.5 \pm 0.7 \mathrm{~S}$ & $6.2 \pm 0.6 \mathrm{~S}$ & $5.6 \pm 0.6 \mathrm{~S}$ \\
\hline HD-5 & $4.7 \pm 0.7 \mathrm{~S}$ & $5.1 \pm 0.9 \mathrm{~S}$ & $2.2 \pm 0.6 \mathrm{~S}$ & $2.9 \pm 0.6 \mathrm{~S}$ & $5.9 \pm 0.4 \mathrm{~S}$ & $4.7 \pm 0.5 \mathrm{~S}$ & $4.9 \pm 0.7 \mathrm{~S}$ & $3.7 \pm 0.5 \mathrm{~S}$ & $3.7 \pm 0.2 \mathrm{~S}$ & $3.8 \pm 1.1 \mathrm{~S}$ & $5.4 \pm 0.4 \mathrm{~S}$ & $5.5 \pm 0.5 \mathrm{~S}$ \\
\hline
\end{tabular}

${ }^{*} \mathrm{R}=$ Resistant, $\mathrm{S}=$ Susceptible

aDisease severity was recorded on a $1-9$ scale on the fourth and fifth inoculated leaves. $1=0 \% ; 2=1-10 \% ; 3=11-20 \% ; 4=21-30 \% ; 5=31-40 \% ; 6=41-50 \% ; 7=51-65 \%$; $8=65-80 \% ; 9=>80 \%$ leaf area covered with lesions.

bMean and standard deviations were calculated from 10 plants.

Table 3: (continued) Pathogenicity and virulence (disease severity on a 1-9 scales) of 25 isolates of Cochliobolus carbonum on 40 corn cultivars. 
Citation: Zhang JX, Yi-Xin Wu, Hon-Hing Ho, Can-Hua Lu, Yue-Qiu He (2013) Cultural Characteristics and Pathogenic Variations among Cochliobolus carbonum Isolates in Yunnan Province of China. J Plant Pathol Microb 4: 210. doi:10.4172/2157-7471.1000210

were s20, s56, s74, s120, s144 and s156, and they were identified as race 2 except for $s 120$.

\section{Discussion}

The cultural characteristics of 25 isolates of C. carbonum from Yunnan were in agreement with those in a previous study [19] and thus their identification was confirmed. Differences between isolates of a pathogen in characters like growth, and sporulation can be critical for determining disease incidence, because these characters help define the fitness of the pathogen. Variation in conidial production has been reported by Welz et al. [17] who showed that race 2 produced significantly more conidia on PLA than race 3. However, no such association was observed in present study. Moreover, there was no clear relationship between the cultural characters and pathogenicity. For example, isolates s44 and s68 were similar morphologically but different in virulence, even though they belonged to the same race. Isolates showed different symptoms on the same inbred line, indicating that there is considerable variation in symptom production by the isolates (Table 2). Conversely, an isolate did not show the same virulence on every cultivar or line. Similarly, Nelson [2] reported that isolates induced different symptoms on the same host and an isolate induced more than one symptom on different hosts.

The occurrence and distribution of physiological races in the field provided important information for the management of northern leaf spot of corn in Yunnan. The information would help to determine, control strategies such as selection and rotation of crop cultivars. In this study, 15 out of 25 isolates belonged to race 3, and 10 belonged to race 2 (Table 2). No isolate of race 1 was recovered from any of the regions surveyed, presumably due to the wide-spread utilization of corn hybrids resistant to race 1 in China. Also, races 0 and 4 were not found, probably due to the small number of maize genotypes susceptible to these races. The findings demonstrated the existence of race 2 and 3 of C. carbonum as the major cause of great losses in maize production in Yunnan Province.

Pathogenic variability of C. carbonum has been demonstrated by Nelson et al. [9], Leonard [6,10], Tsukiboshi et al. [20], Dodd and Hooker [11], Dodd [13], as well as Welz and Leonard [8]. Some of these authors characterized the pathogenic types as races of different cultivars, but differences in virulence of the isolates are little known. Race 3 isolates were considered more virulent than race 2 isolates in the previous studies [21,22]. Other contributing factors might involve the larger and contiguous lesion spots of race 3 and the lack of selection pressure on this race due to the monoculture of corn with a narrow genetic base with most cultivars being susceptible. In this study, most race 3 isolates were more virulent than isolates of race 2 . However, 2 highly virulence strains (s146 and s167) of race 2 were also isolated suggesting that race 2 might also have the potential to cause great damage to corn in Yunnan.

\section{Acknowledgements}

The research was financially supported by the Department of Agriculture, Yunnan Province under the program (TX2009001) of Maize Production System.

\section{References}

1. Shoemaker RA (1959) Nomenclature of Drechslera and Bipolaris, grass parasites segregated from Helminthosporium. Can J Bot 37: 879-887.

2. Nelson RR (1970) Genes for pathogenecity in Cochliobolus carbonum Phytopathology 60: 1335-1337.
3. Welz HG, Leonard KJ (1994) Genetic analysis of two race $0 \times$ race 2 crosses in Cochliobolus carbonum. Phytopathology 84: 83-90.

4. Smith IM, Dunez J, Lelliott RA, Phillips DH, Archer SA (1988) European handbook of plant diseases. Blackwell Scientific Publications, Oxford, UK.

5. Welz HG, Leonard KJ (1995) Gametic phase disequilibria in populations of race 2 and race 3 of Cochliobolus carbonum. Eur J Plant Pathol 101: 301-310.

6. Leonard KJ (1987) The host population as a selective factor. In: Wolfe MS and Caten CE (eds), Populations of Plant Pathogens: Their Dynamics and Their Genetics. Blackwell Scientific Publications, Oxford. 163-179.

7. Welz HG, Leonard KJ (1988) Genetic variation in field population of races 0, 2 and 3 of Bipolaris zeicola in 1987. (Abstr). Phytopathology 78: 1574.

8. Welz HG, Leonard KJ (1993) Phenotypic variation and parasitic fitness of races of Cochliobolus carbonum on corn in North Carolina. Phytopathology 83: 593-601.

9. Nelson RR, Blanco M, Dalmacio S, Moore BS (1973) A new race of Helminthosporium carbonum on corn. Plant Dis Rep 57: 822-823.

10. Leonard KJ (1978) Polymorphisms for lesion type, fungicide tolerance, and mating capacity in Cochliobolus carbonum isolates pathogenic to corn. Can J Bot 56: 1809-1815

11. Dodd JL, Hooker AL (1990) Previously undescribed pathotype of Bipolaris zeicola on corn. Plant Dis 74: 530.

12. Lević J, Penčić V (1992) Response of maize inbred B73 to a new pathotype of Bipolaris zeicola (Stout) Shoemaker. Maydica 37: 355-361.

13. Dodd JL (1993) Recent developments in the maize pathogen Bipolaris zeicola Shoemaker. Maydica 38: 201-204.

14. Tuite J (1969) Plant Pathological Methods, Fungi and Bacteria. Burges Publishing Company, USA

15. Nelson RR (1959) Cochliobolus carbonum, the perfect stage of Helminthosporium carbonum. Phytopathology 49: 807-810.

16. Zhang TY, Sun GY (2000) Flora fungorum sinicorum. (Vol 30), Helminthosporioid Hyphomycetes. Beijing, Science Press.

17. Welz HG, Leath S, Leonard KJ (1993) Sporulation by races 0, 2, and 3 of Cochliobolus carbonum on synthetic medium and sterilized corn leaves. Plant Dis 77: 1153-1157.

18. Stanković S, Lević J, Ivanović D (2007) Genetic variability of maize pathogens in Serbia. Genetika 39: 227-240.

19. Lević J, Penčić V (1993) Morphology of a new pathotype of Bipolaris zeicola (Stout) Shoemaker. J Phytopathol 139: 339-346.

20. Tsukiboshi T, Kimigafukuro T, Sato T (1987) Identification of races of Bipolaris zeicola, the causal fungus of Helminthosporium leaf spot on corn in Japan. Ann Phytopathol Soc Japan 53: 647-649.

21. Castor LL, Ayers JL, Nelson RR, Schreiber AJ (1976) The reaction of several corn inbreeds to race 3 of Helminthosporium carbonum. Plant Dis Rep 60: 827-830.

22. Halseth DE, Pardee WD, Viands DR (1991) Inheritance of resistance to Helminthosporium carbonum race 3 in maize. Crop Sci 31: 612-617. 